\title{
CAPÍTULO 47
}

\section{ESTRATÉGIAS PARA O TRATAMENTO NÃO FARMACOLÓGICO NA SAÚDE DO IDOSO COM DIABETES MELLITUS}

\section{DOI 10.4322/978-65-995353-2-1.c47}

\begin{abstract}
$\underline{\text { Isabella Cristina Galvão da Silva }}{ }^{1}$, Elian Klyscia Miranda Rocha ${ }^{2}$, Antonia Mylene Sousa Almeida ${ }^{3}$, Maria Letícia Gabrielle Feitosa Barbosa ${ }^{4}$, Maria Tailany Sousa Silva ${ }^{5}$, Francisco Lucas Leandro de Sousa ${ }^{6}$, Getúlio Rosa dos Santos Junior ${ }^{7}$, Erika Daniely Vaz de Aquino ${ }^{8}$, Michele Mirley Rodrigues Inácio da Silva9, Brenda Kelly da Silva Monte $^{10}$

${ }^{1}$ Faculdade de Educação São Francisco, (icgds1234@gmail.com) ${ }^{2}$ Faculdade de Educação São Francisco, (klysciamirandaenfer@gmail.com)

${ }^{3}$ Faculdade de Educação São Francisco, (mylenesousa123@hotmail.com)

${ }^{4}$ Faculdade Cesmac do Sertão (leticiagabriellefb@gmail.com)

${ }^{5}$ Faculdade de Integração-FIS (mariatailanyss@ gmail.com) ${ }^{6}$ Centro Universitário Maurício de Nassau (lucasleandro2912@gmail.com)

${ }^{7}$ Universidade Federal do Maranhão (getulio.rosa@ discente.ufma.br) ${ }^{8}$ Centro Universitário Metropolitano da Amazônia (erikavaz2000@gmail.com)

${ }^{9}$ Centro Universitário Cesmac (michelemirley31@ gmail.com)

${ }^{10}$ Mestre em Enfermagem pela Universidade Federal do Piauí UFPI (bksm@faesf.com.br)
\end{abstract}

\section{Resumo}

O objetivo desse estudo é realizar uma revisão da literatura, com a finalidade de identificar quais estratégias podem ser adotadas para a realização do tratamento não farmacológico em pacientes da terceira idade que apresentam diabete mellitus. O presente estudo trata-se de uma revisão literária integrativa, onde o levantamento de artigos se deu pelas bases de dados: Medical Literature Analysis and Retrieval System on-line (MEDLINE via BVS), Literatura Latinio-americana de Ciências da Saúde (LILACS via BVS) e a Base de dados em Enfermagem (BDENF via BVS), a partir dos descritores em ciências da saúde (DeCS): "Saúde do Idoso", "Diabetes Mellitus" e "Cooperação e Adesão ao Tratamento", e os MeSH (Medical Subject Headings): "Health of the Elderly", "Diabetes Mellitus" and "Treatment Adherence and Compliance". Os descritores foram cruzados através do operador booleano "AND" para busca simultânea dos assuntos. Os critérios de inclusão considerados foram os artigos originais disponíveis por meio eletrônico em português ou em inglês, que 
estejam disponíveis no período de 2015 a 2021 por se tratarem de pesquisas atuais. Além disso, os critérios de exclusão foram definidos os artigos duplicados, livros, teses, monografias, revisão, artigos que não tratam da temática proposta e os que apresentam o texto incompleto. Os principais resultados, mostram que alguns idosos tem um conhecimento geral sobre os alimentos que devem evitar/restringir, como por exemplo os produtos ricos em carboidratos e gorduras por serem alimentos que prejudicam a saúde e não fazerem bem ao organismo, principalmente aos pacientes diabéticos. Contudo, esse estudo revela a importância de os idosos adquirirem conhecimentos através da educação em saúde promovida por profissionais capacitados. Através dessa promoção de saúde, essa população passa a conhecer sobre suas comorbidades e as práticas de autocuidado que eles podem realizar.

Palavras-chave: Saúde do Idoso; Diabetes Mellitus; Cooperação e Adesão ao Tratamento. Área Temática: Ciências da saúde

E-mail do autor principal: icgds1234@gmail.com

\section{INTRODUÇÃO}

A diabetes mellitus (DM) se destaca dentre as doenças crônicas não transmissíveis e se caracteriza como um agregado de distúrbios metabólicos definidos por alterações ou defeitos na ação/secreção da insulina, que ocasiona a hiperglicemia, podendo estar relacionada à obesidade e hábitos de vida não saudáveis (BERTONHI; DIAS, 2018).

Existem quatro classificações da DM: tipo 01 ou insulino-dependente (DM1); tipo 02 ou insulino não dependente (DM2); gestacional; e secundário a outras patologias (REIS, 2014). Dentre os tipos citados, a DM tipo 01 corresponde a cerca de $10 \%$ do total dos casos, enquanto a DM tipo 02 está relacionada a 90\% dos casos, sendo essa mais prevalente (BARBOSA; CAMBOIN, 2016).

A DM se estabelece como uma doença que evolui gradualmente, na qual quando acometido, em especial na população idosa, tende a lesar seu estado de saúde com o passar do tempo, principalmente após os 10 anos convivendo com a doença, período que começam a aparecer as complicações provenientes do controle glicêmico inadequado, o que pode refletir na qualidade de vida de forma desfavorável e negativa (LIMA et al., 2018).

Atualmente, o envelhecimento populacional vem crescendo de forma acelerada e devido a isso, há o aparecimento de doenças crônicas incapacitantes que passaram a adquirir uma maior relevância no cenário da saúde pública (SILVA et al., 2016).

Segundo RAMOS et al., (2017), o crescimento da expectativa de vida em idosos, consequentemente reflete em uma maior incidência de doenças crônicas nessa população, especialmente, a DM, isso quando está associado a hábitos de vida inadequados. Ademais, o 
idoso diabético, quando comparado ao não diabético, está mais suscetível a ser medicado abundantemente, exibir carências funcionais devendo ser tratado de forma individualizada e planejada.

A diabete mellitus na população idosa pode ocasionar diversas complicações, como doenças cardiovasculares, retinopatia, nefropatia e neuropatia. Essas complicações podem afetar na qualidade de vida dessa população, em vista disso, há a necessidade de cuidados intensos e atenção dobrada para melhorar a expectativa de vida dos idosos (SILVA et al., 2016).

O tratamento da DM tem a finalidade do controle glicêmico e metabólico, bem como diminuir as complicações associadas a essa doença. Tal tratamento inclui tanto o farmacológico como não farmacológico, podendo citar como exemplos do segundo a prática de exercícios físicos e a dieta especifica e individualizada (BERTONHI; DIAS, 2018).

Com isso, essa temática é de suma importância, pois através dela, pode-se avaliar sobre o método de tratamento não farmacológico para melhorar a qualidade de vida dos idosos. Diante disso, tem-se a seguinte pergunta de pesquisa: de que forma o tratamento não farmacológico pode ser adotado no cotidiano dos idosos com DM? A partir disso, o objetivo desse trabalho é realizar uma revisão da literatura, com a finalidade de identificar quais estratégias podem ser adotadas para a realização do tratamento não farmacológico em pacientes da terceira idade que apresentam diabete mellitus.

\section{MÉTODO}

O presente estudo trata-se de uma revisão literária integrativa, no qual dentre as revisões é o método mais amplo que inclui estudos experimentais ou não para um melhor conhecimento dos estudos considerados. Esse tipo de metodologia pactua também a literatura no âmbito teórico e empírico e tem a finalidade de definir os conceitos, revisar teorias e evidências e analisar problemas metodologicos (SOUZA; DIAS; CARVALHO, 2010).

Ainda de acordo com Souza, Dias e Cervalho (2010), a revisão integrativa é dividida em 06 etapas, sendo elas: a elaboração da pergunta, no qual é a fase mais importante do método; busca da literatura através das bases eletrônicas; coleta de dados; análise dos estudos; argumentação dos resultados; apresentação do método de revisão.

A pergunta norteadora para a pesquisa foi a seguinte: de que forma o tratamento não farmacológico pode ser adotado no cotidiano dos idosos com DM?

O levantamento de artigos se deu pelas bases de dados: Medical Literature Analysis and Retrieval System on-line (MEDLINE via BVS), Literatura Latinio-americana de Ciências da Saúde (LILACS via BVS) e a Base de dados em Enfermagem (BDENF via BVS), a partir dos

\section{E - book Tripé do Ensino Superior: Ensino,} Pesquisa e Extensão 
descritores em ciências da saúde (DeCS): "Saúde do Idoso", "Diabetes Mellitus" e "Cooperação e Adesão ao Tratamento", e os MeSH (Medical Subject Headings): "Health of the Elderly", "Diabetes Mellitus" and "Treatment Adherence and Compliance". Os descritores foram cruzados através do operador booleano "AND” para busca simultânea dos assuntos.

Os critérios de inclusão considerados foram os artigos originais disponíveis por meio eletrônico em português ou em inglês, que estejam disponíveis no período de 2015 a 2021 por se tratarem de pesquisas atuais. Além disso, os critérios de exclusão foram definidos os artigos duplicados, livros, teses, monografias, revisão, artigos que não tratam da temática proposta e os que apresentam o texto incompleto.

\section{RESULTADOS E DISCUSSÃO}

Através do levantamento nas bases de dados, foram encontrados um total de 514 artigos. Dentre eles, 218 foram excluídos por estarem com o texto incompleto, 206 por não corresponder ao período de ano escolhido e 06 por não estarem na linguagem escolhida. Desses, sobraram 84 que foram selecionados para a leitura. Após a análise, 01 foi eliminado pelo método e 62 por não tratar da temática. Diante disso, 21 artigos foram selecionados para o estudo (Figura 1).

Figura 1. Fluxograma de resultados das buscas nas bases de dados, Pedreiras, 2021.

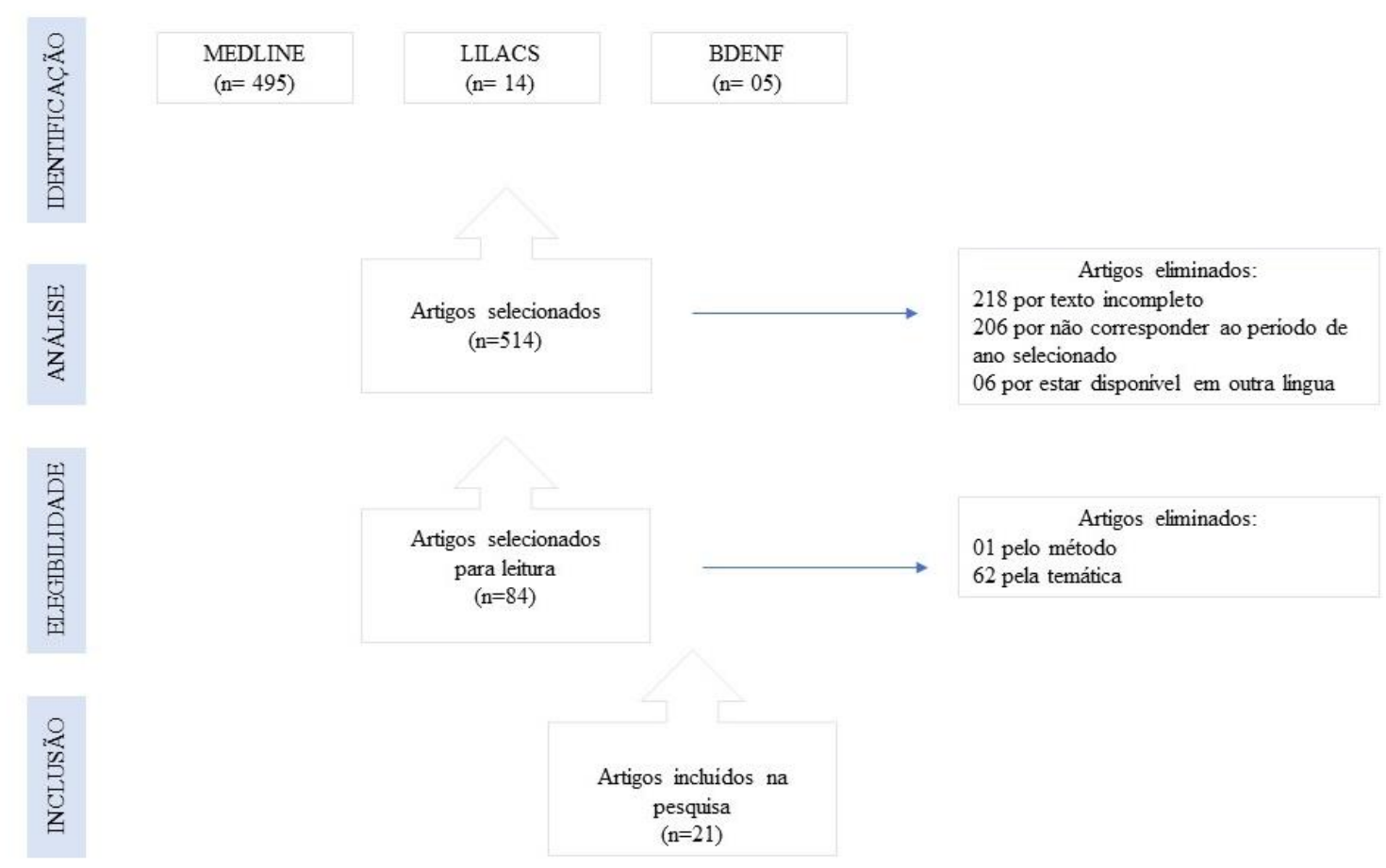


Dentre os anos selecionados para a pesquisa, os que se destacaram foram 2017 e 2018 , considerando uma temática ainda atual, onde os principais objetivos foram associar a educação em saúde com o autocuidado, como também encontrar novas estratégias para melhorar a adesão dos pacientes às recomendações nutricionais.

Os principais resultados, mostram que alguns idosos tem um conhecimento geral sobre os alimentos que devem evitar/restringir, como por exemplo os produtos ricos em carboidratos e gorduras por serem alimentos que prejudicam a saúde e não fazerem bem ao organismo, principalmente aos pacientes diabéticos.

Além disso, estudos apontam também que os pacientes da terceira idade não aderem aos bons hábitos e que os idosos com atitudes negativas tiveram maiores taxas de não adesão às mudanças no estilo de vida. Relatam ainda que a não mudança de padrões alimentares se dá por parte dos demais membros da família que não aderem aos bons hábitos de vida. Alguns pacientes citam o cuidado com os pés e com os ferimentos.

O papel da equipe multiprofissional é importante para identificar os problemas e potencialidades de pessoas idosas com diabetes e seus parentes / cuidadores para garantir adesão às atividades de autocuidado. A formação continuada desses profissionais é extremamente necessária para que eles entendam a importância de aderir a tais atividades e serem capazes de fornecer orientação e conscientização sobre a adoção de hábitos saudáveis (DYONISIO, et al., 2020).

As práticas educativas em grupo são vistas como uma estratégia essencial para a adesão ao tratamento não farmacológico, pois através dessa prática, os usuários adquirem conhecimentos sobre sua comorbidade e aprendem a melhor forma de tratamento, além do farmacológico. Além disso, aumenta a autonomia e capacidade dos idosos para o autocuidado, além de promover sua qualidade de vida (MACEDO et al., 2017).

Ainda de acordo com Macedo, et al. (2017), os profissionais da saúde devem utilizar ferramentas, como as práticas em grupo, para possibilitar aos idosos com DM a aquisição de informações, habilidades e ensinamentos sobre as práticas do autocuidado. A realização de tais atividades é relevante, pois traz benefícios aos indivíduos, podendo citar a melhora nos hábitos alimentares e a prática de exercícios fisicos.

A educação em saúde promovida nas unidades básicas de saúde, é uma forma de disseminar a importância do tratamento não farmacológico aos idosos. Essas informações prestadas a eles, podem ser realizadas de forma oral, através de uma roda de conversa durante a espera para consulta. Outra maneira de passar essas informações é através da escuta ativa, na qual o 
profissional pergunta quais hábitos eles costumam praticar e apartir disso, ensinar sobre os bons hábitos de vida (JARVIE et al., 2019).

O tratamento não farmacológico inclui a prática de atividades físicas, em vista disso, os profissionais da saúde podem realizar um momento de educação associada a prática de exercícios. Essa ação é de suma importancia, pois através dela, pode-se conhecer sobre as formas de tratamento não famacológico e ainda praticar exercícios adequados para os idosos, como por exemplo a dança (JARL et al., 2019).

Os indivíduos na terceira idade tendem a ter dificuldades emocionais e comportamentais, uma vez que perdem ou diminuem o convívio com a sociedade, interferindo assim na sua maneira de se comunicar, pensar e agir, desta forma resulta na resistência ao diálogo com o profissional e principalmente, a seguir o tratamento estabelecido. Cabe a equipe especializada conhecê-lo, se adequar e buscar atendê-lo e trata-lo de acordo com suas necessidades e particularidades (RAMOS, 2017).

O acompanhamento e assistência profissional contínua tornam-se de extrema importância, visto que aumentarão a interação e o vínculo entre o cliente, a família, o profissional e sua equipe multidisciplinar, e desta forma facilitarão a adesão ao tratamento e realização do autocuidado, além de previnir possíveis complicações mais severas que poderão prejudicar de forma mais acentuada a saúde do idoso (ALENCAR, et al., 2017).

Em concordância com Ramos (2017), o desenvolvimento de estratégias educativas de intervenção adaptadas às necessidades e que promovam ao idoso diabético o incentivo ao autocuidado serão possíveis, auxiliando, desta maneira, na promoção da autonomia, prevenção dos agravos e benefícios na qualidade de vida.

\section{CONCLUSÃO}

Contudo, esse estudo revela a importância de os idosos adquirirem conhecimentos através da educação em saúde promovida por profissionais capacitados. Através dessa promoção de saúde, essa população passa a conhecer sobre suas comorbidades e as práticas de autocuidado que eles podem realizar. As informações em saúde são uma estratégia benéfica para o tratamento não farmacológico, pois através dela os idosos podem melhorar sua qualidade de vida por meio de exercícios físicos, boa alimentação e não se limitar apenas ao tratamento farmacológico, previnindo também o uso exacerbado de medicamentos.

Torna-se imprescindível a atuação do grupo familiar na adesão ao tratamento através do apoio emocional, auxiliando as mudanças de estilo de vida e provendo os cuidados básicos com a saúde. Contribuindo com o trabalho dos profissionais em analisar e compreender o usuário

\section{E - book Tripé do Ensino Superior: Ensino,} Pesquisa e Extensão 
não somente como portador de DM, mas sim como indivíduo em todos os seus aspectos e particularidades.

\section{REFERÊNCIAS}

ALENCAR, D. C, et al. Consulta de enfermagem na perspectiva de usuários com diabetes mellitus na estratégia de saúde da família. Revista de Enfermagem UFPE on line. v. 11, n. 10, p. 3749-3756, 2017.

BARBOSA, S. A; CAMBOIN, F. E. F. Diabetes mellitus: cuidados de enfermagem para controle e prevenção de complicações. Temas em saúde. v.16, n.3, 2016.

BERTONHI, L. G; DIAS, J. C. R. Diabetes mellitus tipo 2: aspectos clínicos, tratamento e conduta dioterápica. Revista ciências nutricionais online, v. 2, n. 2, p. 1-10, 2018.

DYONISIO G, et al. Fatores relacionados à adesão às atividades de autocuidado de idosos com diabetes. Bioscience Jornal, v. 36, n. 2, p. 636-651, 2020.

JARL, G. et al. Gender differences in attitudes and attributes of people using therapeutic shoes for diabetic foot complications. Journal of Foot and Ankle Research, v. 12, n. 21, 2019. JARVIE, J. L. et al. Aerobic Fitness and Adherence to Guideline-Recommended Minimum Physical Activity Among Ambulatory Patients With Type 2 Diabetes Mellitus. Diabetes Care, v. 42, July 2019

LIMA, L. R., et al. Qualidade de vida e o tempo do diagnóstico do diabetes mellitus em idosos. Rev. Bras. Geriatr. Gerontol. v.21, n.2, p.180-190, 2018.

MACEDO M. M. L, et al. Adesão e empoderamento de usuários com diabetes mellitus para práticas de autocuidado: ensaio clínico randomizado. Rev Esc Enferm USP, v.51: e03278, 2017

RAMOS, R. S. P. S., et al. Fatores associados ao diabetes em idosos assistidos em serviço ambulatorial especializado geronto-geriátrico. Rev. Bras. Geriatr. Gerontol., v.20, n.3, Pesquisa e Extensão 
p.364-374, 2017.

REIS, T. B. Tratamento não - farmacológico do diabetes mellitus. 2014.

SILVA, A. B, et al. Prevalência de diabetes mellitus e adesão medicamentosa em idosos da Estratégia Saúde da Família de Porto Alegre/RS. Cad. Saúde Colet., Rio de Janeiro, v. 24, n. 3, p. 308-316, 2016.

SOUZA, M. T; SILVA, M. D; CARVALHO, R. Revisão integrativa: o que é e como fazer. Einstein., v. 8, n. 1, p. 102-6, 2010. 